\title{
Histone methyltransferase G9a contributes to H3K27 methylation in vivo
}

Cell Research (2011) 21: 365-367. doi:10.1038/cr.2010.157; published online 16 November 2010

\section{Dear Editor,}

Histone modifications play a vital role in the conformation and function of their associated chromatin templates [1]. Histone H3K27 methylation mediated by the PRC2 complex is critical for transcriptional regulation, Polycomb silencing, Drosophila segmentation, mammalian $\mathrm{X}$ inactivation and cancer [1]. Interestingly, H3K27me1 (H3 mono-methylated at residue K27) levels in vivo remain unaffected after PRC2 disruption [2, 3], which is an indication for the existence of other contributing histone methyltransferase(s) to H3K27me1. However, in animals, histone methyltransferases responsible for H3K27me1 levels in vivo remain undefined. On the other hand, G9a is a histone methyltransferase that controls $\mathrm{H} 3 \mathrm{~K} 9 \mathrm{me} 2$ in vivo and predominantly represses genes at euchromatic regions [4]. It has also been reported to act on $\mathrm{H} 3 \mathrm{~K} 27$ in vitro on $\mathrm{H} 3$ tail fused with a GST tag [5], but its role in H3K27 methylation has not been tested on the nucleosomes, the native substrates for histone methyltransferases. In addition, G9a's contribution to H3K27 methylation in vivo remains obscure.

We previously reported that histones containing methyl-lysine analogues (MLA) [6] are compatible in biochemical reactions mediated by many histone methyltransferases, including Dot1L, HYPB, Suvar4-20 and PrSet7 [7]. We then extended this study to G9a. His-tagged $\mathrm{H} 3$ histones containing K9C mutation were converted into His-H3Kc9me2 with the MLA reaction to mimic homogenously di-methylated H3K9. To our surprise, recombinant G9a displayed robust activity on nucleosomes assembled with H3Kc9me2 (Figure 1A, left lane), which suggests that either G9a may be a trimethylase or may react with other $\mathrm{H} 3$ lysine residues on a nucleosomal substrate. Thus, we introduced a K27A mutation into the His-tagged pre-methylated $\mathrm{H} 3$, which nearly completely abolished G9a's activity (Figure 1A, right lane), indicating that G9a is mainly a dimethylase and also reacts with H3K27.

To directly detect the reaction products, wild-type nucleosomes were reacted with G9a and S-adenosyl methionine. The reaction products were chemically propionylated [8] prior to mass spectrometry analysis to ensure the detection of $\mathrm{H} 3 \mathrm{~K} 9$ methylation marks in subsequent LC-MS (liquid chromatograph-coupled mass spectrometry) procedures. G9a-treated nucleosomal H3 samples contained $\mathrm{H} 3 \mathrm{~K} 9 \mathrm{me} 1, \mathrm{H} 3 \mathrm{~K} 9 \mathrm{me} 2$ signals and low amounts of $\mathrm{H} 3 \mathrm{~K} 9 \mathrm{me} 3$ as expected (Supplementary information, Figure S1). Moreover, they also contained robust signals for H3K27me1 and H3K27me2 (Figure 1B). In addition, another histone methyltransferase GLP, a close homologue of G9a, can also methylate H3K27 in addition to H3K9 in vitro (Supplementary information, Figure S2).

The role of G9a as an $\mathrm{H} 3 \mathrm{~K} 9$ methyltransferase has been fully recognized [4], but its contribution to H3K27 methylation in vivo remains obscure. This is likely due to the overwhelming contribution of PRC2 in H3K27 methylation $[2,3]$, which may mask the contribution of G9a. Methylation status-specific antibodies were used to compare the levels of H3K27 methylation between wildtype and G9a ${ }^{-/}$ES cells. Although no apparent changes of H3K27me2/3 levels were observed, H3K27me1 levels were clearly reduced in the G9a ${ }^{-/-}$ES cells (Figure 1C).

To quantitatively compare the histone modification levels between the wild-type and G9a ${ }^{-/-}$ES cells, stable isotope labeling-based quantitative mass spectrometry analysis $[9,10]$ was performed. Histone samples were prepared from wild-type ES cells fully labeled with lysine-8 $\left(\left[{ }^{13} \mathrm{C}_{6},{ }^{15} \mathrm{~N}_{2}\right]\right.$ heavy isotope-labeled L-lysine, abbreviated as K8, Supplementary information, Figure S3) and then mixed with equal amounts of histones extracted from G9a ${ }^{-/-}$ES cells labeled with regular L-lysine (K0). $\mathrm{H} 3 \mathrm{~K} 27 \mathrm{me} 1$ levels in the $\mathrm{G}^{-a^{-/}}$ES cells were reduced to about $70 \%$ of the wild-type levels (Figure 1D and 1E). Comparison of H3K27me2 levels between wild-type and $\mathrm{G}^{-} \mathrm{a}^{-/-}$ES cells revealed a more complicated and interesting pattern. G9a ${ }^{-/-}$ES cells contained similar levels (97\%) of $\mathrm{H} 3$ carrying the combination of K27me2 and K36me2, reduced levels $(75 \%)$ of $\mathrm{H} 3$ carrying the combination of K27me2 and K36me1, and further reduced levels (47\%) of $\mathrm{H} 3$ carrying the combination of K27me 2 and $\mathrm{K} 36 \mathrm{me} 0$ 


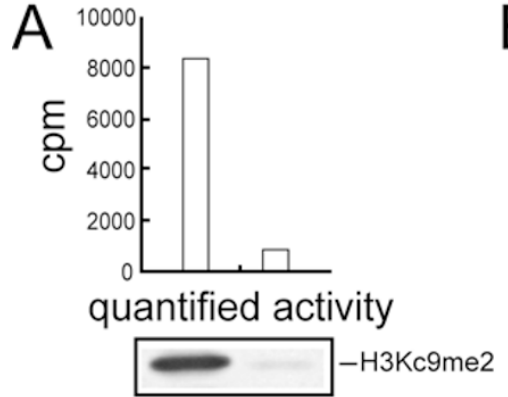

autoradiography

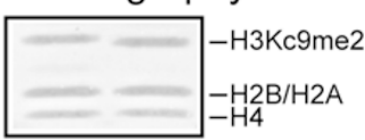

commassie

Lys Ala $\mathrm{H} 3$ residue 27
B

K(pro-me1)SAPATGGVK(pro)K(pro)PHR

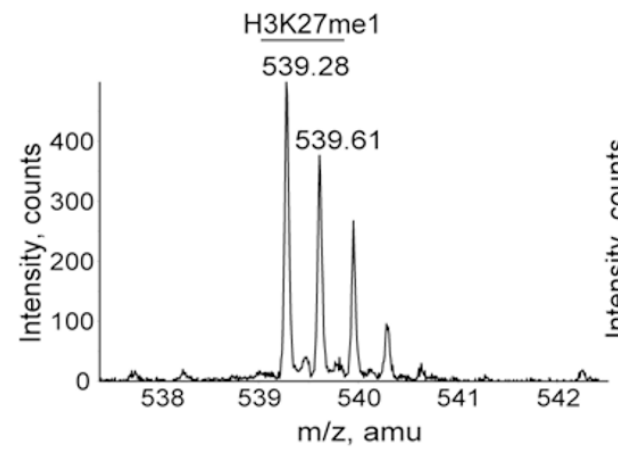

pro: propionylation
H3K27me1

H3K27me1

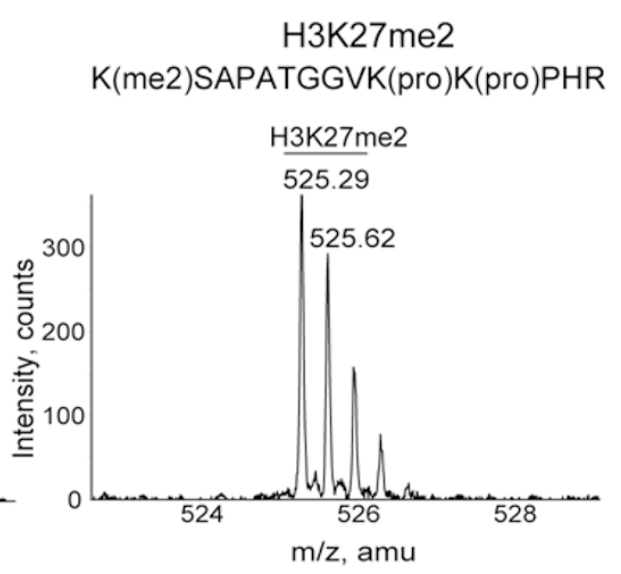

C
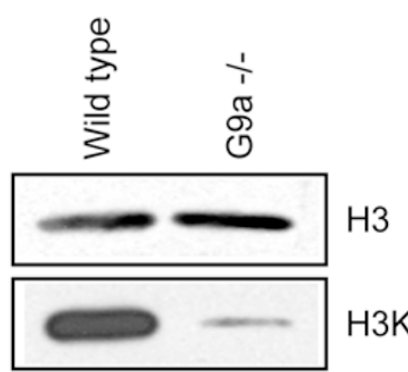

H3K9me2
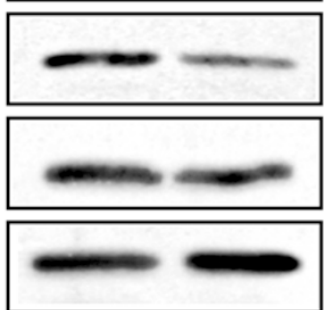

H3K27me2

H3K27me3

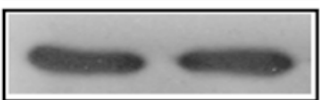

Actin

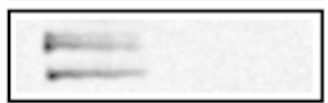

G9a

Western

E

$\mathrm{H} 3$ back bone $\mathrm{K}$ (pro)LPFQR, $2 \mathrm{H}^{+}, \mathrm{K} 0 / \mathrm{K} 8=0.86$

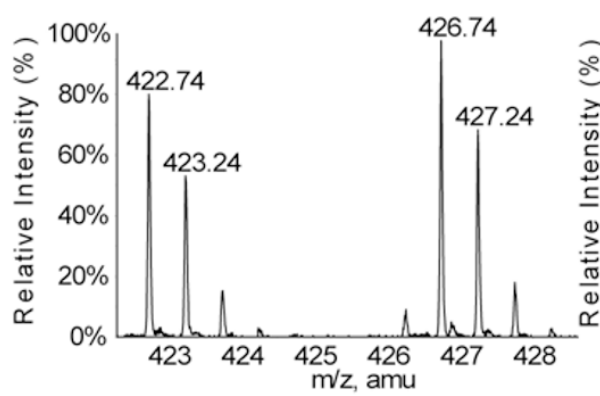

D

$\frac{\mathrm{K} 0 / \mathrm{K} 8\left(\mathrm{G}_{9} \mathrm{a}^{-/-} / \text {Wild type }\right)}{\text { raw ratio normalized ratio }}$

H3 back bone

VTIMPK(pro)DIQLAR $\quad 0.85$

K(pro)LPFQR

0.86

H3K27me0

K(pro)SAPATGGVK(pro)K(pro)PHR

0.91

1.06

H3K27me1

K(pro-me1)SAPATGGVK(pro)K(pro)PHR $\quad 0.61 \quad 0.71$

K(pro-me1)SAPATGGVK(pro-me1)K(pro)PHR $0.63 \quad 0.74$

H3K27me2

$\begin{array}{lll}\text { K(me2)SAPATGGVK(pro)K(pro)PHR } & 0.40 & 0.47\end{array}$

K(me2)SAPATGGVK(pro-me1)K(pro)PHR $0.64 \quad 0.75$

$\begin{array}{lll}\text { K(me2)SAPATGGVK(me2)K(pro)PHR } & 0.83 & 0.97\end{array}$

H3K27me3

K(me3)SAPATGGVK(pro-me1)K(pro)PHR $\quad 0.91 \quad 1.06$

H3K9me2

K(me2)STGGK(pro)APR

0.23

0.27
H3K27me2

K(pro-me1)SAPATGGVK(pro)K(pro)PHR, K(me2)SAPATGGVK(pro)K(pro)PHR, $3 \mathrm{H}^{+}, \mathrm{KO} / \mathrm{K} 8=0.63$ $3 \mathrm{H}^{+}, \mathrm{KO} / \mathrm{K} 8=0.40$
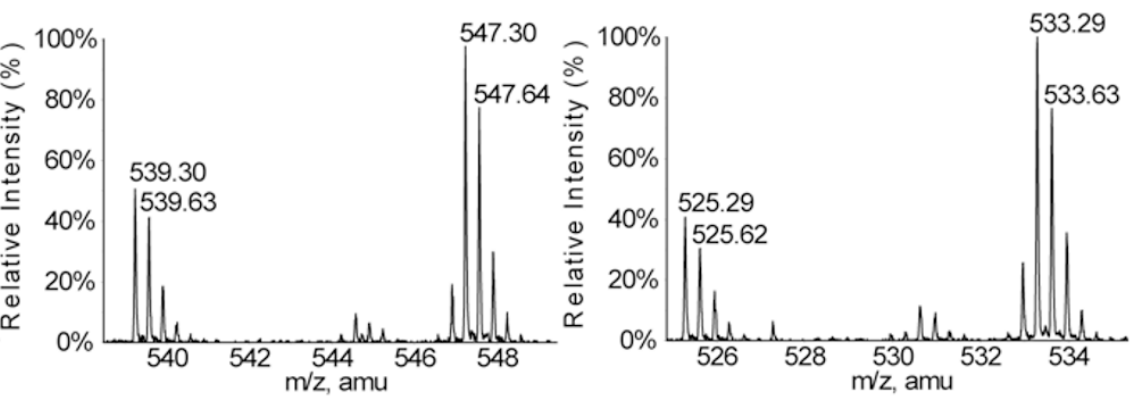
Figure $1 \mathrm{G} 9 \mathrm{a}$ methylates H3K27 in vitro and contributes to H3K27 methylation in vivo. (A) G9a methylates nucleosomal $\mathrm{H} 3 \mathrm{~K} 27$ in vitro; (B) direct detection of $\mathrm{H} 3 \mathrm{~K} 27 \mathrm{me} 1$ and $\mathrm{H} 3 \mathrm{~K} 27 \mathrm{me} 2$ products by mass spectrometry on nucleosomal $\mathrm{H} 3$ reacted with G9a; (C) $\mathrm{G9a}^{-/-}$ES cells displayed reduced H3K27me1 levels in vivo; (D) stable isotope labeling-based quantitative mass spectrometry revealed reduced levels of $\mathrm{H} 3 \mathrm{~K} 27 \mathrm{me} 1$ and a subset of $\mathrm{H} 3 \mathrm{~K} 27 \mathrm{me} 2$ in G9a ${ }^{-/-}$ES cells.

(Figure 1D and 1E, Supplementary information, Figure S4). These results suggest a potential cross-talk between H3K27 and H3K36 methylations.

Taken together, our results collectively suggest G9a's contribution to $\mathrm{H} 3 \mathrm{~K} 27$ methylation in addition to its well-characterized role in $\mathrm{H} 3 \mathrm{~K} 9$ methylation in vivo.

PRC2 complexes containing Ezh2 and its closely related Ezh1 have been clearly shown to be the main contributors for $\mathrm{H} 3 \mathrm{~K} 27 \mathrm{me} 2 / 3$ in vivo [3, 4]. Identification of G9a and possibly GLP as novel contributors to H3K27me1 and H3K27me2 in vivo led to an intriguing question for further investigation: do G9a/GLP coordinate $\mathrm{H} 3 \mathrm{~K} 9$ and $\mathrm{K} 27$ methylation at certain chromatin regions for a specific function?

\section{Acknowledgments}

We are grateful to Y Shinkai at Kyoto University for providing the G9a null ES cells and cDNA. This work was supported by the Chinese Ministry of Science and Technology 863 projects 2007AA02Z1A6 (to BZ) and 2007AA02Z1A3 (to SC).

Hui $\mathrm{Wu}^{1,2}$, Xiuzhen Chen ${ }^{2,3}$, Jun Xiong ${ }^{1,2}$, Yingfeng $\mathrm{Li}^{2,3}$, Hong $\mathrm{Li}^{2}$, Xiaojun Ding ${ }^{2}$, Sheng Liu ${ }^{2}$, She Chen ${ }^{2}$, Shaorong $\mathrm{Gao}^{2}$, Bing $\mathrm{Zhu}^{2}$

${ }^{I}$ Graduate Program, Peking Union Medical College and Chinese Academy of Medical Sciences, Beijing 100730, China; ${ }^{2}$ National Institute of Biological Sciences, Beijing 102206, China; ${ }^{3}$ Life Science College, Beijing Normal University, Beijing 100875, China

Correspondence: Bing Zhu

Tel: +86-10-80728458; Fax: +86-10-80715648

E-mail: zhubing@nibs.ac.cn

\section{References}

1 Allis CD, Jenuwein T, Reinberg D. In: Allis CD, Jenuwein T, Reinberg D, eds. Epigenetics. New York: Cold Spring Harbor Laboratory Press, 2006:23-56.

2 Cao R, Zhang Y. SUZ12 is required for both the histone methyltransferase activity and the silencing function of the EEDEZH2 complex. Mol Cell 2004; 15:57-67.

3 Pasini D, Bracken AP, Jensen MR, et al. Suz12 is essential for mouse development and for EZH2 histone methyltransferase activity. EMBO J 2004; 23:4061-4071.

4 Tachibana M, Sugimoto K, Nozaki M, et al. G9a histone methyltransferase plays a dominant role in euchromatic histone $\mathrm{H} 3$ lysine 9 methylation and is essential for early embryogenesis. Genes Dev 2004; 16:1779-1791.

5 Tachibana M, Sugimoto K, Fukushima T, Shinkai Y. Set domain-containing protein, G9a, is a novel lysine-preferring mammalian histone methyltransferase with hyperactivity and specific selectivity to lysines 9 and 27 of histone H3. J Biol Chem 2001; 276:25309-25317.

6 Simon MD, Chu F, Racki LR, et al. The site-specific installation of methyl-lysine analogs into recombinant histones. Cell 2007; 128:1003-1012.

7 Jia G, Wang W, Li H, et al. A systematic evaluation of the compatibility of histones containing methyl-lysine analogues with biochemical reactions. Cell Res 2009; 19:1217-1220.

8 Garcia BA, Mollah S, Ueberheide BM, et al. Chemical derivatization of histones for facilitated analysis by mass spectrometry. Nat Protoc 2007; 2:933-938.

9 Ong SE, Blagoev B, Kratchmarova I, et al. Stable isotope labeling by amino acids in cell culture, SILAC, as a simple and accurate approach to expression proteomics. Mol Cell Proteomics 2002; 1:376-386.

$10 \mathrm{Xu}$ M, Long C, Chen X, Huang C, Chen S, Zhu B. Partitioning of histone $\mathrm{H} 3-\mathrm{H} 4$ tetramers during DNA replication-dependent chromatin assembly. Science 2010; 328:94-98.

(Supplementary information is linked to the online version of the paper on the Cell Research website.) 